\title{
Autoimmune Hemolytic Anemia Confers an Independent Risk Factor for Thrombosis: Retrospective Cohort Study Using the "STRIDE" Database
}

\author{
Chen EC ${ }^{1}$, Loftus PD ${ }^{1}$, Weber SC ${ }^{1}$, Hoang NM, \\ Gilbert J ${ }^{2}$, Rosenthal $\mathbf{A}^{2}$ and Kummar $\mathrm{S}^{1 *}$ \\ ${ }^{1}$ Stanford School of Medicine, Stanford University, USA \\ ${ }^{2}$ True North Therapeutics, USA
}

*Corresponding author: Shivaani Kummar, Stanford University School of Medicine, 780 Welch Road, CJ 250L, Palo Alto, CA 94305, USA

Received: May 07, 2017; Accepted: J une 12, 2017; Published: July 04, 2017

\begin{abstract}
Background: Auto Immune Hemolytic Anemia (AIHA) is a rare autoimmune disorder in which auto antibodies cause haemolysis. While thrombosis is considered a complication of AIHA, whether AlHA confers an independent thrombosis risk has not been thoroughly shown. The relationship between haemolysis and thrombosis is also unclear.
\end{abstract}

Methods: 312 non-AlHA patients from Stanford University's clinical database were matched 2:1 with 156 AlHA patients. Thrombosis incidence was measured, and the prevalence of other thrombosis risk factors as defined by the PADUA score, prior splenectomy status, antiphospholipid antibody diagnosis, and concomitant anticoagulation use was also compared. Within AlHA patients, the relationship between thrombosis and haemolysis was analyzed in terms of time and severity.

Results: $29 \%$ of AlHA patients developed thrombosis compared to $19 \%$ of non-AlHA patients $(p<0.05)$. AlHA conferred an odds ratio of $2.44(95 \% \mathrm{Cl}$ [1.16-5.10], $p<0.05)$ for thrombosis. The median PADUA score was not different between the two groups (4 for AIHA, IQR [3-7] vs. 4.5 for non-AIHA, IQR [37], n.s.). There was also no difference in prior splenectomy, antiphospholipid antibody status, and concomitant anticoagulation use. AlHA patients with thrombosis had more hemolytic flares than patients without thrombosis $(24.5$ instances vs. 13.8, respectively; $\mathrm{p}<0.05$ ) and a higher lifetime drop in hemoglobin $(53.4 \mathrm{~g} / \mathrm{dL}$ vs. $27.1 \mathrm{~g} / \mathrm{dL}$, respectively; $\mathrm{p}<0.05) .81 \%$ of patients with thrombosis had the event within one week of a hemolytic flare.

Conclusion: AlHA is an independent risk factor for thrombosis. Thrombosis is associated with periods of haemolysis and a high hemolytic burden.

Keywords: Anemia; Autoimmune; Hemolytic; Thrombosis; Thromboembolism

\section{Introduction}

Auto Immune Hemolytic Anemia (AIHA) is a rare autoimmune disorder in which auto antibodies target red blood cell surface antigens, causing haemolysis. The estimated incidence is 0.8 per 100,000 with a prevalence of 17 per 100,000 [1,2]. Depending on the thermal range at which the auto antibodies are most active, AIHA is classified as warm (wAIHA), cold (cold agglutinin disease, or CAD), or mixed. 50-60\% of AIHA cases are believed to be secondary [3-5] Major risk factors for secondary AIHA include malignancy [6], viral and mycoplasma infections [7,8], and rheumatologic disorders [9]. AIHA treatment remains largely based on expert opinion without a clear consensus due to limited prospective data $[10,11]$. Treatment is focused on immune suppression, cytotoxic agents, splenectomy, and addressing contributing factors [12].

While thromboembolism is widely considered to be a complication of AIHA, few studies have assessed whether AIHA confers an independent thrombosis risk that cannot be attributed to known thrombosis risk factors such as immobilization, malignancy, and rheumatologic disorders. Doing so requires the comparison of thrombosis rates in matched AIHA and non-AIHA patients, where other thrombosis risk factors can be taken into account. The literature on this topic is summarized by a 2015 meta-analysis by Ungprasert, et al. [13] based on three retrospective cohort studies and one cross-sectional study. This meta-analysis arrived at an overall pooled risk ratio of 2.63 (95\% CI [1.37-5.05]) for the development of thromboembolism in AIHA [13]. However, the studies included the meta-analysis were limited in the extent to which they matched their AIHA and non-AIHA cohorts. Yusuf, et al. matched their non-AIHA cohort according to age and gender alone [14], while the other three studies required their non-AIHA cohort to only lack a diagnosis of AIHA [15-17].

Within AIHA patients, the relationship between thrombosis and haemolysis is also not well understood. Haemolysis is generally thought to cause thromboembolism due to the abnormal exposure of phosphatidylserine following red cell destruction, which promotes coagulation [18]. In support, work by Barcellini, et al. [11] and Lecouffe-Desprets, et al. have suggested that thrombosis in
Ann Hematol Oncol - Volume 4 Issue 7 - 2017 ISSN : 2375-7965 | www.austinpublishinggroup.com Kummar et al. @ All rights are reserved
Citation: Chen EC, Loftus PD, Weber SC, Hoang NM, Gilbert J, Rosenthal A, et al. Autoimmune Hemolytic Anemia Confers an Independent Risk Factor for Thrombosis: Retrospective Cohort Study Using the "STRIDE" Database. Ann Hematol Oncol. 2017; 4(7): 1160. 
Table 1: Characteristics of AlHA and non-AlHA cohorts.

\begin{tabular}{|c|c|c|c|}
\hline & $\begin{array}{c}\text { AlHA } \\
(\mathrm{N}=156)\end{array}$ & $\begin{array}{l}\text { Non-AIHA } \\
(\mathrm{N}=312)\end{array}$ & $\begin{array}{c}\text { p-value } \\
\text { ("denotes } p<0.05 \text { ) }\end{array}$ \\
\hline \multicolumn{4}{|l|}{ Demographic } \\
\hline \multicolumn{4}{|l|}{ Gender } \\
\hline Male, N (\%) & $60(38 \%)$ & $122(39 \%)$ & 0.8343 \\
\hline Female, N (\%) & $96(62 \%)$ & $190(61 \%)$ & 0.8343 \\
\hline Age, Mean (SD) & $60.0(19.3)$ & $60.3(18.6)$ & \\
\hline Smoking, N (\%) & $40(26 \%)$ & $74(24 \%)$ & 0.6365 \\
\hline \multicolumn{4}{|l|}{ Major AIHA risk factors } \\
\hline Malignancy visits, Median (IQR) & $1(1-2)$ & $1(1-2)$ & 0.9681 \\
\hline Rheumatologic disorder, N (\%) & $74(47 \%)$ & $148(47 \%)$ & 1.0000 \\
\hline Viral/mycoplasma infection, $\mathrm{N}(\%)$ & $45(29 \%)$ & $90(29 \%)$ & 1.0000 \\
\hline Patients with thrombosis, N (\%) & $45(29 \%)$ & $58(19 \%)$ & 0.0144 \\
\hline
\end{tabular}

AIHA: Auto Immune Hemolytic Anemia; N: Number; IQR: Interquartile Range

AIHA is associated with more severe anemia at the time of AIHA onset and at the time of the thrombotic event $[19,20]$. However, these studies excluded patients with secondary causes of AIHAi.e., lymphoproliferative disorders, infections, and autoimmune disorders-which comprise the majority of AIHA cases as mentioned above.

Thus, our objectives were (1) to ascertain the risk of thrombosis intrinsic to AIHA that cannot be attributed to other major thrombosis risk factors, and (2) to better characterize the relationship between haemolysis and thrombosis within AIHA. The strength of this study is the use of a longitudinal, retrospective cohort design with propensity score matching between AIHA and non-AIHA patients.

\section{Methods}

\section{Study population}

STRIDE (Stanford Translational Research Integrated Database Environment) is a standards-based informatics platform supporting clinical and translational research at Stanford University [21]. The STRIDE database was initiated in 2005 and houses records for over
2.6 million patients who have received care at Stanford University Medical Center since 1995. Our study cohort was derived from all available records in STRIDE database from 1995 until November 2015. Data was obtained following all applicable institutional and ethics approvals.

The inclusion criteria were as follows: (1) age $\geq 18$, (2) at least one instance of hemoglobin (hgb) $\leq 12 \mathrm{~g} / \mathrm{dL}$, (3) at least one instance of haptoglobin $<8 \mathrm{mg} / \mathrm{dL}$, (4) diagnosis of AIHA as defined by a positive direct antiglobulin test (DAT), and (5) documentation of the ICD9 code for AIHA (283.0). Unlike prior studies, patients were not excluded based on secondary causes of AIHA such as hematologic, neoplastic, or infectious processes. A total of 156 AIHA patients met the above criteria in the STRIDE database and were classified into AIHA subtypes: wAIHA (DAT+ for IgG without evidence of clinically significant cold agglutinins), CAD (DAT + for C3 only with high thermal amplitude cold agglutinins) [22], and mixed (DAT+ for both IgG and C3 with high thermal amplitude cold agglutinins). DAT positivity for $\mathrm{C} 3$ but without follow-up thermal amplitude tests were deemed unclassifiable.

To assess the risk of thromboembolism conferred by AIHA, an age and gender-matched control cohort of non-AIHA patients was derived for comparison. 312 non-AIHA patients were matched with the 156 AIHA patients in a 2:1 ratio. Patients in the non-AIHA group were prioritized to match according to the major risk factors for AIHA: malignancy (where the severity of active disease was estimated by the number of cancer center visits), viral and mycoplasma infections, and rheumatologic diseases. To prioritize matching according to AIHA risk factors, 6 non-AIHA patients were matched by removing the gender constraint, and 8 were matched by removing both the gender and age constraints.

Within the AIHA and non-AIHA cohort, the association between thrombosis and common thrombosis risk factors, based on the PADUA criteria, was determined. The PADUA criteria were originally developed as a tool for assessing the risk factors for venous thromboemboli (VTEs) [23]. These factors include active malignancy, previous thromboembolism, reduced mobility, existing

Table 2: PADUA risk factors for AIHA and non-AlHA patients with thrombosis.

\begin{tabular}{|c|c|c|c|}
\hline & $\begin{array}{l}\text { AlHA with thrombosis } \\
\qquad(\mathrm{N}=45)\end{array}$ & $\begin{array}{l}\text { Non-AIHA with thrombosis } \\
\qquad(\mathrm{N}=58)\end{array}$ & $\begin{array}{c}\text { p-value } \\
\text { ("denotes } p<0.05)\end{array}$ \\
\hline \multicolumn{4}{|l|}{ PADUA risk factors } \\
\hline Active malignancy, N (\%) & $12(27 \%)$ & $32(55 \%)$ & 0.0046 \\
\hline Previous DVT, N (\%) & $11(24 \%)$ & $3(5.2 \%)$ & 0.0057 \\
\hline Reduced mobility, N (\%) & $17(38 \%)$ & $16(28 \%)$ & 0.2843 \\
\hline Thrombophilic condition, N (\%) & $6(13 \%)$ & $12(21 \%)$ & 0.2916 \\
\hline Recent ( $\leq 1$ month) trauma or surgery, N (\%) & $16(36 \%)$ & $13(22 \%)$ & 0.1188 \\
\hline Age $\geq 70, N(\%)$ & $8(18 \%)$ & $11(19 \%)$ & 0.8975 \\
\hline Heart or respiratory failure, $\mathrm{N}(\%)$ & $10(22 \%)$ & $3(5.2 \%)$ & 0.0110 \\
\hline Prior MI or stroke, N (\%) & $7(16 \%)$ & $5(8.6 \%)$ & 0.2511 \\
\hline Acute infection or rheumatologic disorder, N (\%) & $17(38 \%)$ & $12(21 \%)$ & 0.0590 \\
\hline Ongoing hormonal therapy, N (\%) & $5(11 \%)$ & $8(14 \%)$ & 0.6516 \\
\hline PADUA score, Median (IQR) & $4(3-7)$ & $4.5(3-7)$ & 0.8729 \\
\hline
\end{tabular}

AlHA: Auto Immune Hemolytic Anemia; N: Number; DVT: Deep Venous Thrombosis; MI: Myocardial Infarction; BMI: Body Mass Index; IQR: Inter Quartile Range 
Table 3: Antiphospholipid antibody diagnosis, prior splenectomy, and concomitant anticoagulation for AIHA and non-AIHA patients with thrombosis.

\begin{tabular}{|l|c|c|l|}
\hline & $\begin{array}{c}\text { AlHA with } \\
\text { thrombosis } \\
(\mathbf{N}=45)\end{array}$ & $\begin{array}{c}\text { Non-AIHA with } \\
\text { thrombosis } \\
(\mathbf{N}=58)\end{array}$ & p-value \\
\hline $\begin{array}{l}\text { Antiphospholipid antibodies, } \\
\text { N (\%) }\end{array}$ & $4(8.9 \%)$ & $1(1.7 \%)$ & 0.0930 \\
\hline $\begin{array}{l}\text { Prior splenectomy (>1 month), } \\
\text { N (\%) }\end{array}$ & $3(6.7 \%)$ & $1(1.7 \%)$ & 0.1949 \\
\hline $\begin{array}{l}\text { Concomitant anticoagulation, } \\
\text { N (\%) }\end{array}$ & $6(13 \%)$ & $8(14 \%)$ & 0.6970 \\
\hline
\end{tabular}

AlHA: Autoimmune Hemolytic Anemia; N: Number

thrombophilic condition (e.g. Factor V Leiden, my eloproliferative disorders), recent trauma or surgery ( $\leq 1$ month), elderly age ( $\geq 70$ years), cardiovascular and/or respiratory failure, myocardial infarctions and/or strokes, obesity (BMI $\geq 30$ ), rheumatologic disorder and/or infection, and hormonal treatment (e.g. hormone replacement therapy, oral contraceptives). The PADUA risk factors comprise a weighted sum known as the PADUA score (max of 20), where scores $\geq 4$ are considered high-risk for VTEs.

The PADUA score omits two additional major thrombotic risk factors, antiphospholipid antibodies and history of splenectomy, and these were considered separately. Patients were considered to be antiphospholipid antibody positive if they were documented as such in clinical notes. Antiphospholipid positivity was not based on lab results since diagnosis requires positive test results 12 weeks apart, and the STRIDE database lacks repeat lab results for work-up initiated during a patient's presentation for AIHA. For splenectomies, only splenectomies performed $>1$ month from a thrombotic event was considered, since thrombotic complications soon after splenectomy are more likely due to local surgical factors [24], which are already captured by the PADUA criteria (i.e., "recent trauma or surgery").

Patients were considered to have been on anticoagulation at the time of their thrombotic event if there was (1) documented use of subcutaneous heparin or enoxaparin within 3 days of their documented date of thrombosis, or (2) documented use of oral agents (e.g. warfarin, rivaroxaban, apixaban) any time prior to thrombosis, since oral agents are typically warranted by long-term indications and were presumed to have been continued through the time of thrombosis unless noted otherwise.

To assess the relationship between thrombosis and haemolysis within the AIHA group, haemolysis laboratory values of patients with and without thrombosis were compared. These labs included hemoglobin (hgb), lactate dehydrogenase (LDH), haptoglobin, and total bilirubin (TBili) levels. Indirect bilirubin levels, though preferred over TBili levels for haemolysis, were not documented in STRIDE for many patients and therefore could not be used.

\section{Statistical analysis}

The incidence of arterial and venous thromboembolism in the AIHA and non-AIHA cohorts was compared using the Student's $\mathrm{t}$-Test (for brevity, "thrombosis" is used in this article interchangeably with "thromboembolism").

In our study, the median PADUA score between AIHA and non-AIHA patients with thrombosis was compared using the Mann-Whitney rank sum test. Because the PADUA score requires calculation at the time of a sentinel event, for patients with thrombosis the score was calculated at the time of thrombosis, or, for AIHA-patients without thrombosis, calculated at the time of AIHA diagnosis. PADUA scores were unable to be calculated for non-AIHA patients without thrombosis.

In seeking to derive an odds ratio of thrombosis given a diagnosis of AIHA, propensity score-matching was used to ensure stringent matching. The propensity score was constructed using patient characteristics (i.e. age, gender, AIHA risk factors, and PADUA risk factors) for each patient, and indicated their likelihood of having AIHA. Inverse-probability weighting was applied on a final model where thrombosis-status was the outcome and AIHA-status was the primary predictor [25]. Using this full model, an odds ratio was derived for the development of thrombosis given a diagnosis of AIHA. To ensure that the model was not over-fitted and the relationship between AIHA and thrombosis was not over-adjusted, a sensitivity analysis was conducted using a sparse model in which only AIHA and covariates significant at $\mathrm{p}<0.05$ were included.

Table 4: PADUA risk factors for AlHA patients with and without thrombosis.

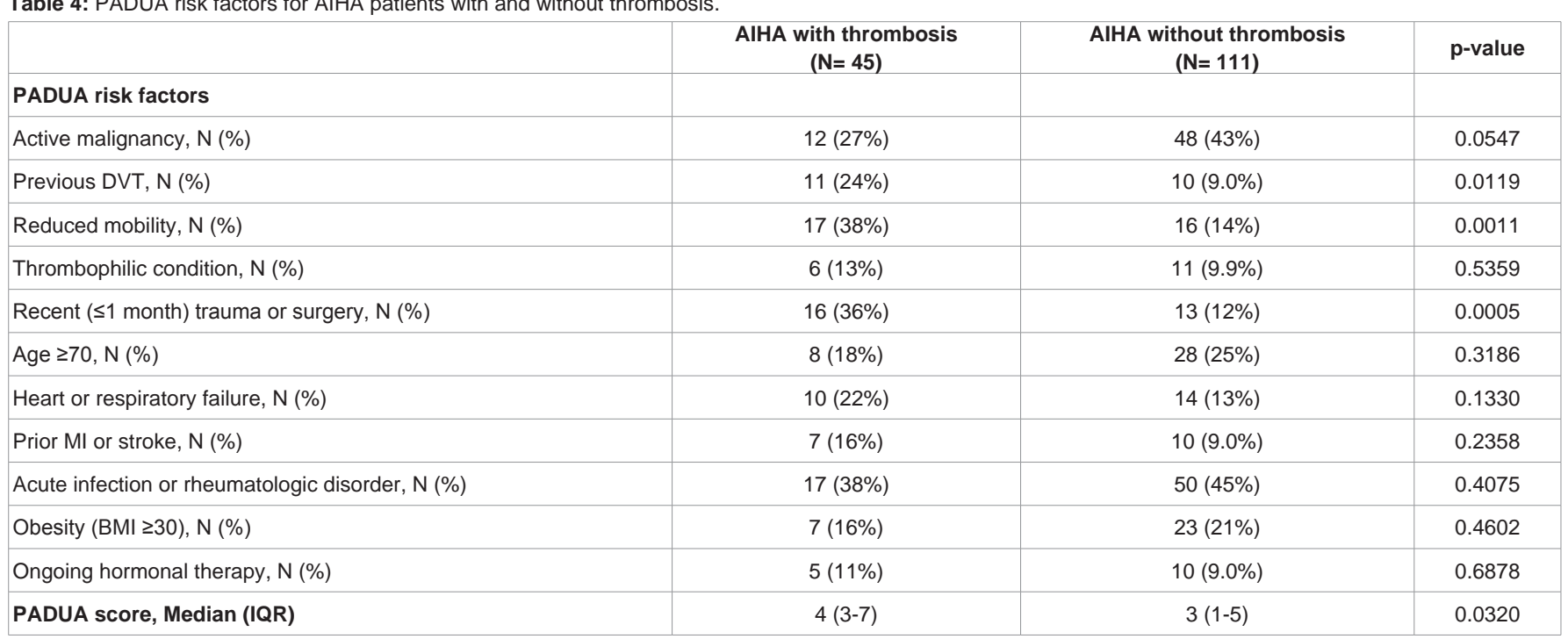

AIHA: Autoimmune Hemolytic Anemia; N: Number; DVT: Deep Venous Thrombosis; MI: Myocardial Infarction; BMI: Body Mass Index; IQR: Interquartile Range 
Table 5: Relationship between thrombosis and haemolysis in AlHA patients with and without thrombosis.

\begin{tabular}{|c|c|c|c|}
\hline & $\begin{array}{l}\text { AlHA with thrombosis } \\
\qquad(\mathrm{N}=45)\end{array}$ & $\begin{array}{l}\text { AlHA without thrombosis } \\
\qquad(\mathrm{N}=111)\end{array}$ & p-value \\
\hline $\mathrm{Hgb}<8 \mathrm{~g} / \mathrm{dL}, \mathrm{N}(\%)$ & $25(55.6 \%)$ & $51(45.9 \%)$ & 0.2737 \\
\hline LDH >340 IU/L, N (\%) & $30(67 \%)$ & $67(60 \%)$ & 0.4159 \\
\hline TBili>3.0 mg/dL, N (\%) & $19(42 \%)$ & $33(30 \%)$ & 0.1514 \\
\hline Average number of hemolytic flares ( $\geq 2 \mathrm{~g} / \mathrm{dL} /$ week), \# of flares & 24.5 & 13.8 & 0.0072 \\
\hline Average number of transfusions, $\mathrm{N}(\%)$ & 24.7 & 21.8 & 0.6705 \\
\hline
\end{tabular}

AlHA: Autoimmune Hemolytic Anemia; N: Number. Hgb: Hemoglobin; LDH: Lactate Dehydrogenase; TBili: Total Bilirubin.

In the event of haemolysis, hgb and haptoglobina are expected to be low with associated high levels of LDH and TBili. Stanford laboratories cutoffs of LDH $>340 \mathrm{IU} / \mathrm{L}$ and haptoglobin $<8 \mathrm{mg} /$ $\mathrm{dL}$ were used to indicate clinically significant positive and negative results, respectively. Cutoffs of hgb $<8 \mathrm{~g} / \mathrm{dL}$ and TBili $>3.0 \mathrm{mg} /$ $\mathrm{dL}$ were chosen according to the Common Terminology Criteria for Adverse Events (CTCAE) for grade 3 (i.e., "severe or medically significant") anemia and hyperbilirubinemia.

The number of haemolysis flares per patient, defined as any period of continuous hgb decline $\geq 2 \mathrm{~g} / \mathrm{dL}$ week, and patients' total lifetime drop in hgb (the "hemolytic burden") were also assessed. Lastly, whether thrombotic events occurred closely in time to hemolytic flares was determined. Differences were compared using the Student's t-Test or two-proportion t-Test.

\section{Results}

\section{AlHA and non-AlHA cohort characteristics}

Demographic and clinical characteristics of patients in the AIHA and non-AIHA cohorts are shown in Table 1. Of the AIHA patients that were classifiable into AIHA subtypes, the majority were diagnosed with wAIHA (44\%), followed by CAD (19\%), mixed (6\%), and unclassifiable (31\%). A significantly higher proportion of AIHA patients developed thrombosis than non-AIHA patients ( $29 \% v s$. $19 \%$, respectively; $\mathrm{p}<0.05)$.

Demographic and risk factor characteristics of the thrombosis and no-thrombosis subgroups within the AIHA and non-AIHA cohorts are shown in Supplemental Table 1. In the AIHA group, thrombosis was associated with a higher frequency of rheumatologic disorders (62\%) and viral/mycoplasma infections (42\%) compared to patients without thrombosis ( $41 \%$ and $23 \%$, respectively; $\mathrm{p}<0.05$ ). In the non-AIHA group, thrombosis was more associated with female gender (69\%) and rheumatologic disorders (62\%) compared to patients without thrombosis ( $41 \%$ and $44 \%$, respectively; $\mathrm{p}<0.05$ ).

\section{Comparing AIHA and non-AlHA patients with thrombosis}

The frequency of PADUA risk factors in AIHA and non-AIHA patients with thrombosis are shown in Table 2. Despite a difference in the incidence of thrombosis in the AIHA and non-AIHA cohort as previously mentioned, the median PADUA score was not different between thrombosis patients in the AIHA and group (4, interquartile range (IQR) (3-7) vs. 4.5, IQR (3-7) respectively; n.s). The difference in the frequency of antiphospholipid antibody diagnosis, prior splenectomies, or concomitant use of anticoagulation at the time of thrombosis between AIHA and non-AIHA patients also did not reach statistical significance (Table 3). The distribution of PADUA risk factors did differ between the two groups. Malignancy was seen in a smaller proportion of AIHA patients with thrombosis than in non-AIHA counterparts ( $27 \%$ vs. $55 \%$, respectively; $\mathrm{p}<0.05)$, while AIHA patients with thrombosis had a higher proportion of patients with prior DVTs $(24 \%$ vs. $5.2 \%$, respectively; $\mathrm{p}<0.05)$ and heart or respiratory failure ( $22 \%$ vs. $5.2 \%$, respectively; $\mathrm{p}<0.05)$.

Supplemental Table 2 shows that AIHA and non-AIHA patients with thrombosis do not significantly differ in the types of thromboembolism that develop, though the $\mathrm{n}$ is small for each comparison. Among patients with thrombosis in either cohort, extremity deep venous thrombus (DVT) are the most prevalent (51\% in AIHA and 55\% in non-AIHA; n.s.), followed by pulmonary embolism (29\% in AIHA and 19\% in non-AIHA; n.s.).

Within the AIHA cohort, patients with thrombosis had a higher total PADUA score than patients without thrombosis, as expected. As seen in Table 4, AIHA patients with thrombosis had a higher proportion of patients with prior DVTs ( $24 \%$ vs. $9 \%$, respectively; $\mathrm{p}<0.05$ ), reduced mobility ( $38 \%$ vs. $14 \%$; respectively, $\mathrm{p}<0.05$ ), and recent trauma and/or surgery ( $36 \%$ vs. $12 \%$, respectively; $\mathrm{p}<0.05)$ than AIHA patients without thrombosis.

Supplemental Table 3 shows that propensity scores for the AIHA and non-AIHA patients differed despite age and risk factor based matching. Inverse probability weighting was used in the final model where outcome is thrombosis-status and main predictor is AIHAstatus (see Methods section). The result of this analysis showed that AIHA confers an odds ratio of 2.44 (95\% CI [1.16-5.10]; $\mathrm{p}=0.018$ ) for the development of thrombosis.

\section{Characterizing the relationship between thrombosis and haemolysis in AlHA patients}

Prior studies have shown that thrombosis is correlated with low hemoglobin at the time of AIHA onset and laboratory evidence of haemolysis $[19,20]$. We did not find these to be the case for our AIHA cohort. AIHA patients with thrombosis did not, at the time of AIHA diagnosis have lower hgb and haptoglobin and higher LDH and TBili compared to patients without thrombosis (Table 5).

It has previously been showed that AIHA patients exhibited lower hgb levels at the time of their thrombotic event, suggesting that periods of severe haemolysis-or "hemolytic flares"-may be a risk factor for thrombosis [20]. We assessed this further by devising two metrics, "hemolytic flares" and total "hemolytic burden" (see Methods section for details). Of our 45 AIHA patients with thrombosis, $91 \%$ of 
the patients had at least one hemolytic flare, and of these patients for whom the time of thrombosis was captured in STRIDE ( $\mathrm{N}=36), 81 \%$ were found to have had a thrombotic event within one week from a flare. Overall, AIHA patients with thrombosis had more hemolytic flares than AIHA patients without thrombosis (24.5 instances vs. 13.8, respectively; $\mathrm{p}<0.05$; Table 5). Moreover, AIHA patients exhibited a higher hemolytic burden compared to patients without thrombosis (i.e., a lifetime Hgb drop of $53.4 \mathrm{~g} / \mathrm{dL}$ vs. $27.1 \mathrm{~g} / \mathrm{dL}$, respectively; $\mathrm{p}<$ 0.05 ; Table 5)

\section{Discussion}

To our knowledge, this is the first retrospective cohort study that compares the incidence and risk factors of thrombosis in AIHA patients, inclusive of secondary AIHA patients, with a closely matched cohort of non-AIHA patients. Prior studies have been smaller in size or descriptive $[5,26,27]$, done in the context of specific hyper coagulable states $[28,29]$, or with a design that excluded secondary AIHA patients or lacked anon-AIHA cohort matched for relevant AIHA risk factors [14-17,19,20].

Logistic regression with propensity score analysis was used to assess the association between AIHA and thrombosis. This crosssectional method was used instead of more traditional survival analysis methods such as Cox proportional hazards modeling because we believed that the proportional hazards assumption would not be met due to right-censoring in the AIHA group. Furthermore, "time to thrombosis" was not possible to be determined in the non-AIHA group due to lack of clear start dates. Our results confirm that AIHA patients have a higher incidence of thrombosis (29\%) than non-AIHA patients (19\%). This thrombosis incidence in AIHA patients is on the higher end of present estimates that include $11 \%$ [19], 20\% [20], and $27 \%$ [29]. This may be due to the enrichment for more medically complex patients at Stanford Hospital and Clinics. Importantly, the PADUA score between the non-AIHA and AIHA patients were not significantly different in our study. Neither did the two cohorts differ in their rates of antiphospholipid antibody diagnosis, prior splenectomy, or concomitant anticoagulant use. These data suggest that the increased thrombosis risk in AIHA patients cannot be attributed to other common thrombosis risk factors. Instead, the observed thrombosis risk appears to be intrinsic to the diagnosis of AIHA.

Ultimately, our analysis showed that AIHA confers an odds ratio of 2.44 (95\% CI [1.16-5.10],) for the development of thromboembolism, which is less than the hazard ratio of 6.30 (95\% CI [4.44-8.94]) reported by the most recent retrospective study on this subject by Yusuf, et al. in 2015 [14]. Methodological differences may explain the difference. In Yusuf, et al., the non-AIHA group was matched by only gender and age. Since the risk factors for secondary AIHA, such as active malignancy, are associated with their own increased risk of thrombosis, we matched AIHA and non-AIHA according to major AIHA risk factors and their overall propensity score for developing AIHA. Interestingly, our odds ratio is similar to the pooled risk ratio of 2.63 (95\% CI [1.37-5.05]) obtained by Ungprasert, et al.'s meta-analysis [13]. Our study appears to strengthen the findings of previous studies.

We find the relationship between thrombosis and haemolysis in AIHA to be also compelling. Lecouffe-Desprets, et al. previously suggested that unprovoked venous thromboemboli are associated with lower hemoglobin levels at the time of the thrombotic event [20]. We found that $91 \%$ of AIHA patients with thrombosis experienced hemolytic flares in their lifetime; moreover, $81 \%$ of these patients with a recorded date of thrombosis experienced the thrombotic event within one week of a hemolytic flare. We recognized that the number of hemolytic flares may be falsely elevated by blood transfusionsi.e., a transfusion may interrupt one continuous flare and generate what appears to be two separate flares. To help circumvent this, the average number of transfusions for AIHA patients with and without haemolysis was also assessed, along with the patients' "hemolytic burden". The later metric should be unaffected by the number of red cell transfusions received since properly cross-matched transfusions should not fuel haemolysis. Overall, AIHA patients with thrombosis experienced more episodes of hemolytic flares and endured a higher lifetime burden of haemolysis than their counterparts without thrombosis. We are hopeful that both metrics-"hemolytic flare" and "hemolytic burden"-will help clinicians portend periods of increased thrombotic risks in their patients. Clinicians may readily calculate, or obtain a quick gestalt for, both metrics using the ability of electronic medical records systems to aggregate longitudinal data.

Our findings add AIHA to the growing list of hemolytic anemia's whose association with coagulopathy are increasingly recognized (e.g. beta-thalassemia, paroxysmal nocturnal hemoglobinuria, sickle cell disease) [30]. Several mechanisms for thrombosis during haemolysis have been proposed, including the abnormal exposure of phosphatidylserine following red blood cell destruction, which may make erythrocytes more adhesive and promote activation of coagulation pathways $[18,30]$. Others have suggested that haemolysis leads to nitric oxide depletion by free plasma hemoglobin, as well as abnormal erythrocyte-endothelium interactions that lead to elevated levels of tissue factor on vessel endothelium [30,31].

This close association between haemolysis and thrombosis in AIHA suggests that thrombo-prevention may be achieved by minimizing periods of hemolytic flares. Shi, et al. Recently showed that a novel serine protease $\mathrm{C} 1 \mathrm{~s}$ inhibitor could inhibit complement activation by cold agglutinins, the main mechanism of haemolysis in cold agglutinin disease [32]. If flares are unable to be prevented, general anticoagulation may be considered. In a small audit study of 28 patients, Hendricks found that anticoagulation in the form of unfractionated heparin, tinzaparin, and coumadin led to fewer instances of thromboembolism when given to hospitalized patients until their haemolysis stabilized [26]. Aside from haemolysis, other thrombosis risk factors to consider include history of prior DVTs, immobility, and recent surgery or trauma, as shown in Table 4.

A limitation of our study is that STRIDE does not capture care that patients may have received elsewhere, nor does it capture reasons for loss of follow-up (e.g. patient death versus achieving disease remission). Consequently, our assessment of a patient's trend of haemolysis and overall hemolytic burden may be underestimations. Additionally, we acknowledge that our patients' initial clinical presentation of AIHA may not correspond to their date of diagnosis in STRIDE, which can be typical for tertiary referral centers like Stanford. This may explain why we found that AIHA patients 
with thrombosis did not have more severe laboratory evidence of haemolysis at the time of their diagnosis at Stanford than those without thrombosis, in contrast to prior studies [19]. Being a tertiary center, Stanford's patient population is also likely to have more severe disease. Finally, despite efforts to control for several possible confounders between AIHA and non-AIHA patients, it is possible that residual confounding remains.

In summary, our result suggests AIHA confersa thrombosis risk that is not attributable to other common thrombosis risk factors. Our results also showed that thrombosis in AIHA is closely associated with hemolytic flares, both temporally and with respect to a patient's overall lifetime burden of haemolysis. Future studies are needed to determine the optimal strategy of thrombo-prevention for this rare disease.

\section{References}

1. Klein NP, Ray P, Carpenter D, Hansen J, Lewis E, Fireman B, et al. Rates of autoimmune diseases in Kaiser Permanente for use in vaccine adverse event safety studies. Vaccine. 2010; 28: 1062-1068.

2. Eaton WW, Rose NR, Kalaydjian A, Pedersen MG, Mortensen PB Epidemiology of autoimmune diseases in Denmark. J Autoimmun. 2007; 29: $1-9$

3. Baek SW, Lee MW, Ryu HW, Lee KS, Song IC, Lee HJ, et al. Clinical features and outcomes of autoimmune hemolytic anemia: a retrospective analysis of 32 cases. Korean J Hematol. 2011; 46: 111-117.

4. Sokol RJ, Hewitt S, Stamps BK. Autoimmune haemolysis: an 18-year study of 865 cases referred to a regional transfusion centre. Br Med J (Clin Res Ed). 1981; 282: 2023-2027.

5. Roumier M, Loustau V, Guillaud C, Languille L, Mahevas M, Khellaf M, et al. Characteristics and outcome of warm autoimmune hemolytic anemia in adults: New insights based on a single-center experience with 60 patients. Am J Hematol. 2014; 89: E150-5.

6. Puthenparambil J, Lechner K, Kornek G. Autoimmune hemolytic anemia as a paraneoplastic phenomenon in solid tumors: A critical analysis of 52 cases reported in the literature. Wien Klin Wochenschr. 2010; 122: 229-236.

7. Saif MW. HIV-associated autoimmune hemolytic anemia: an update. AIDS Patient Care STDS. 2001; 15: 217-224.

8. Khan FY, A yassin M. Mycoplasma pneumoniae associated with severe autoimmune hemolytic anemia: case report and literature review. Braz J Infect Dis. 2009; 13: 77-79

9. Giannouli S, Voulgarelis M, Ziakas PD, Tzioufas AG. Anaemia in systemic lupus erythematosus: from pathophysiology to clinical assessment. Ann Rheum Dis. 2006; 65: 144-148.

10. Lechner K, Jager U. How I treat autoimmune hemolytic anemias in adults Blood. 2010; 116: 1831-188.

11. Zanella A, Barcellini W. Treatment of autoimmune hemolytic anemias. Haematologica. 2014; 99: 1547-1554.

12. Bass GF, Tuscano ET, Tuscano JM. Diagnosis and classification of autoimmune hemolytic anemia. Autoimmun Rev. 2014; 13: 560-564.

13. Ungprasert $P$, Tanratana $P$, Srivali N. Autoimmune hemolytic anemia and venous thromboembolism: A systematic review and meta-analysis. Thromb Res. 2015; 136: 1013-1017

14. Yusuf HR, Hooper WC, Grosse SD, Parker CS, Boulet SL, Ortel TL. Risk of venous thromboembolism occurrence among adults with selected autoimmune diseases: a study among a U.S. cohort of commercial insurance enrollees. Thromb Res. 2015; 135: 50-57.
15. Zoller B, Li X, Sundquist J, Sundquist K. Risk of pulmonary embolism in patients with autoimmune disorders: a nationwide follow-up study from Sweden. Lancet. 2012; 379: 244-249.

16. Ramagopalan SV, Wotton CJ, Handel AE, Yeates D, Goldacre MJ. Risk of venous thromboembolism in people admitted to hospital with selected immune-mediated diseases: record-linkage study. BMC Med. 2011; 90: 1-7015-9-1.

17. Yusuf HR, Hooper WC, Beckman MG, Zhang QC, Tsai J, Ortel TL. Risk of venous thromboembolism among hospitalizations of adults with selected autoimmune diseases. J Thromb Thrombolysis. 2014; 38: 306-313.

18. Ataga KI. Hypercoagulability and thrombotic complications in hemolytic anemias. Haematologica. 2009; 94: 1481-1484.

19. Barcellini W, Fattizzo B, Zaninoni A, Radice T, Nichele I, Di Bona E, et al. Clinical heterogeneity and predictors of outcome in primary autoimmune hemolytic anemia: a GIMEMA study of 308 patients. Blood. 2014; 124: 29302936.

20. Lecouffe-Desprets M, Neel A, Graveleau J, Leux C, Perrin F, Visomblain B, et al. Venous thromboembolism related to warm autoimmune hemolytic anemia: a case-control study. Autoimmun Rev. 2015; 14: 1023-1028.

21. Lowe $\mathrm{HJ}$, Ferris TA, Hernandez PM, Weber SC. STRIDE--An integrated standards-based translational research informatics platform. AMIA Annu Symp Proc. 2009; 2009: 391-395.

22. Swiecicki PL, Hegerova LT, Gertz MA. Cold agglutinin disease. Blood. 2013 ; 122: $1114-1121$.

23. Barbar S, Noventa F, Rossetto V, Ferrari A, Brandolin B, Perlati M, et al. A risk assessment model for the identification of hospitalized medical patients at risk for venous thromboembolism: the Padua Prediction Score. J Thromb Haemost. 2010; 8: 2450-2457.

24. Crary SE, Buchanan GR. Vascular complications after splenectomy for hematologic disorders. Blood. 2009; 114: 2861-2868.

25. Curtis LH, Hammill BG, Eisenstein EL, Kramer JM, Anstrom KJ. Using inverse probability-weighted estimators in comparative effectiveness analyses with observational databases. Med Care. 2007; 45: S103-7.

26. Hendrick AM. Auto-immune haemolytic anaemia--a high-risk disorder for thromboembolism? Hematology. 2003; 8: 53-56.

27. Klein MK, Dow SW, Rosychuk RA. Pulmonary thromboembolism associated with immune-mediated hemolytic anemia in dogs: ten cases (1982-1987). J Am Vet Med Assoc. 1989; 195: 246-250.

28. Kokori SI, Ioannidis JP, Voulgarelis M, Tzioufas AG, Moutsopoulos HM Autoimmune hemolytic anemia in patients with systemic lupus erythematosus. Am J Med. 2000; 108: 198-204.

29. Pullarkat V, Ngo M, Iqbal S, Espina B, Liebman HA. Detection of lupus anticoagulant identifies patients with autoimmune haemolytic anaemia at increased risk for venous thromboembolism. Br J Haematol. 2002; 118: 1166 1169.

30. Cappellini MD. Coagulation in the pathophysiology of hemolytic anemias Hematology Am Soc Hematol Educ Program. 2007: 74-78.

31. Rother RP, Bell L, Hillmen P, Gladwin MT. The clinical sequelae of intravascular hemolysis and extracellular plasma hemoglobin: a novel mechanism of human disease. JAMA. 2005; 293: 1653-1662.

32. Shi J, Rose EL, Singh A, Hussain S, Stagliano NE, Parry GC, et al. TNT003, an inhibitor of the serine protease $\mathrm{C} 1 \mathrm{~s}$, prevents complement activation induced by cold agglutinins. Blood. 2014; 123: 4015-4022.
Ann Hematol Oncol - Volume 4 Issue 7 - 2017 ISSN : 2375-7965 | www.austinpublishinggroup.com Kummar et al. () All rights are reserved
Citation: Chen EC, Loftus PD, Weber SC, Hoang NM, Gilbert J, Rosenthal A, et al. Autoimmune Hemolytic Database. Ann Hematol Oncol. 2017; 4(7): 1160 